\title{
Flow cytometry in cell-based pharmacokinetics or cellular kinetics in adoptive cell therapy
}

\author{
Vellalore N Kakkanaiah*,1, Kevin R Lang ${ }^{1}$ \& Patrick K Bennett ${ }^{1}$ \\ ${ }^{1}$ PPD Laboratories, 2244 Dabney Road, Richmond, VA 23230, USA \\ *Author for correspondence: Vellalore.Kakkanaiah@ppdi.com
}

First draft submitted: 26 July 2018; Accepted for publication: 12 August 2018; Published online: 14 September 2018

Pharmacokinetics (PK) defines the disposition of a drug in the body based primarily on measurements in fluids or tissues by various bioanalytical methods during the drug development. Most bioanalytical methods utilize ligandbinding or LC-MS technology, and the assays are validated and applied following the guidelines from various regulatory agencies in their countries (e.g., US FDA, EMEA or Health Canada) [1]. These bioanalytical method guidelines focus on the PK of traditional drug compounds; small molecules and large biomolecules.

The CAR-T (chimeric antigen receptor) cell therapies, recently approved by FDA, involve administration of 'living drugs' capable of proliferation after infusion. This behavior is very different from conventional drug compounds, and the term 'cellular kinetics' was coined for in vivo 'PK monitoring' of both the expansion and persistence of the genetically engineered CAR-T cells [2]. The bioanalytical methods used to measure the levels of these infused cells also differ from conventional methodologies; molecular (polymerase chain reaction-qPCR and sequencing) and cellular assays (flow cytometry) [3]. These two technologies play a significant role in the drug development in the recent years. This article is focused on the challenges in the development of flow cytometry bioanalytical methods to quantitatively measure CAR-T cell levels in adoptive cell therapy.

\section{Flow cytometry methods}

The flow cytometer is unique for its ability to measure multiple parameters including cell surface markers and intracellular molecules on individual cells at a high rate. Flow cytometry has been used extensively as a clinical diagnostic tool for leukemia phenotyping, measuring CD4 T-cell counts in HIV samples and other diagnostic assays. Clinical diagnostic methods must follow CLSI guidelines for method validation [4]. Recently, flow cytometry methods are employed in all stages of drug development [5] and established regulatory guidelines for method validation and application do not address this technology. However, the pharmaceutical scientific community has published White Papers for the validation of flow cytometry methods [6. Most flow cytometry assays are considered biomarker assays, and the methods are typically developed and validated as fit-for-purpose to measure safety and pharmacodynamics (PD) biomarkers, including target engagement and receptor occupancy, especially when humanized antibodies against cell surface receptors are used. Method validation for PK studies by flow cytometry was also described when using antibody drugs that bind to cell surface receptors [7]. These methods are focused on small and large molecules that are chemically well defined. But the genetically engineered cells in adoptive cell therapy are heterogeneous with increased diversity on a single cell basis [8]. Therefore, it is very crucial to measure the 'purity' of the cells, which will reflect the potency of the cellular therapy.

\section{Method development challenges for CAR-T enumeration}

In CAR T-cell therapy, autologous (patient) or allogeneic (donor) $T$ cells are purified, genetically engineered to express receptors against tumor-specific surface antigens and expanded in the laboratory. These expanded cells are reinfused into the cancer patient, where they bind to tumor cell surface antigens in non-MHC restricted fashion, proliferate and kill the tumor cells. The measurement of infused CAR-T cell kinetics is vital to evaluate the in vivo post-antigen exposure related expansion and subsequence persistence. Based on the recent data from various clinical trials on CAR-T cell therapy, the expansion of the infused CAR-T cells peaks within the 2 weeks following 
infusion and declines over a period of months. Even after years, detectable levels of CAR-T cells are present in vivo [9].

The first challenge for method development is acquiring samples (e.g., whole blood, bone marrow, CSF) with CAR-T cells. In autologous CAR-T cell therapy, each patient receives their own T cells, obtaining patient's samples before therapy is not possible. Therefore, CAR-T cells need to be manufactured using healthy donor samples with the same CAR molecules. These donor CAR-T cells can then be spiked into healthy donor samples and used for method development. Since the CAR-T cell manufacturing is complex and expensive, it adds to the burden on drug development.

A second challenge is the sample stability. Unlike plasma or serum sample analysis, where the samples are frozen for later sample analysis, the freezing of whole blood, bone marrow, CSF samples for cellular flow cytometry analysis is not possible in most cases. Therefore, the samples need to be processed and analyzed with a very limited stability period usually within $2-3$ days.

Third, very few reagents are commercially available against CAR molecules on the T-cell surfaces, such as CD19-Ig-fusion protein [10], adding additional time and expense to the sponsor or the laboratory to generate and characterize the reagents against CAR molecule of interest.

\section{Regulatory challenges}

While PK requirements are well described in recent FDA guidance for bioanalytical method validation, a minimal guidance is included for biomarker method validation. It states that biomarker assays should be fully validated when the biomarker data are used for regulatory decision making, such as safety, potency and dosing [11]. Flow cytometry methods differ from ligand-binding assay and other biomarker assays where some of the validation parameters are not applicable, such as accuracy and specificity where these parameters should be verified during the development of the assay [6]. In addition, the requirement of incurred sample reanalysis for PK method validation is another challenge for flow cytometry method due to short sample stability and varying cellular expression. Therefore, the differences in the various technology platforms used for PK method validations need clear guidance from the regulatory agencies.

\section{Conclusion}

Adoptive cell therapy for cancer and infections with certain viruses presents additional challenges for bioanalytical laboratories to develop reliable sensitive methods to study the 'cellular kinetics' of the infused T cells. At present, qPCR and flow cytometry methods are employed to quantify the persisting infused T cells. Recent advances in the flow cytometry to detect multiple markers simultaneously made this method well-suited to study both phenotype and functional characteristics of these $\mathrm{T}$ cells. However, due to lack of cellular reference material and complex instrumentation, the validation of the flow cytometry methods is more challenging compared with other established methods, such as ligand-binding assay or MS. Therefore, consensus among pharmaceutical industry, bioanalytical laboratories and regulatory agencies is vital to establish guidelines for the flow cytometry method validation.

\section{Financial \& competing interests disclosure}

The authors have no relevant affiliations or financial involvement with any organization or entity with a financial interest in or financial conflict with the subject matter or materials discussed in the manuscript. This includes employment, consultancies, honoraria, stock ownership or options, expert testimony, grants or patents received or pending, or royalties.

No writing assistance was utilized in the production of this manuscript.

\section{References}

1. Arnold M, Fluhler E, Gorovits B. Understanding bioanalysis regulations. In: Regulated Bioanalysis: Fundamentals and Practice. AAPS Advances in the Pharmaceutical Sciences Series 26. Rocci ML Jr, Lowes S (Eds). Springer International Publishing AG Cham, Switzerland (2017).

2. Mueller KT, Maude SL, Porter DL et al. Cellular kinetics of CTL019 in relapsed/refractory B-cell acute lymphoblastic leukemia and chronic lymphocytic leukemia. Blood 130(21), 2317-2325 (2017).

3. Kalos M. Biomarkers in T cell therapy in clinical trials. J. Transl. Med. 9,138 (2011).

4. Clinical and Laboratory Standards Institute. Enumeration of Immunologically Defined Cell Populations by Flow Cytometry; Approved Guideline(2nd Edition). CLSI document H42-A2. Clinical and Laboratory Standards Institute, PA, USA (2007).

5. Litwin V, Marder P. Flow Cytometry in Drug Discovery and Development. John Wiley \& Sons, Inc., NJ, USA (2011). 
6. O’Hara DM, Xu Y, Liang Z, Reddy MP, Wu DY, Litwin V. Recommendations for the validation of flow cytometric testing during drug development: II assays. J. Immunol. Methods. 363(2), 120-134 (2011).

7. Xu Y, Richards SM. Pharmacokinetics by flow cytometry: recommendations for development and validation of flow cytometric method for pharmacokinetic studies. Flow Cytometry in Drug Discovery and Development, Chapter 11. Litwin V, Marder P (Eds). John Wiley \& Sons, Inc., NJ, USA (2011).

8. Milone MC, Bhoj VG. The pharmacology of T cell therapies. Mol. Ther. Methods Clin. Dev. 8, 210-221 (2018).

9. Scholler J, Brady TL, Binder-Scholl G et al. Decade-long safety and function of retroviral-modified chimeric antigen receptor T cells. Sci. Transl. Med. 4, 132ra53 (2012).

10. De Oliveira SN, Wang J, Ryan C, Morrison SL, Kohn DB, Hollis RP. A CD19/Fc fusion protein for detection of anti-CD19 chimeric antigen receptors. J. Transl. Med. 11, 23 (2013).

11. US FDA. Guidance for Industry: Bioanalytical Method

Validation (2018). https://www.fda.gov/downloads/drugs/guidances/ucm070107.Pdf 
